\title{
A Saving Energy Routing Protocol in WSN Network
}

\author{
Yang Wenzhong, Zhang Zhenyu \\ School of Information Science and Engineering Xinjiang University \\ URUMQI 830046, China \\ E-mial: ywz_xy@163.com
}

\begin{abstract}
Currently, energy-efficient routing protocol is a research hot topic in WSN networks due to the limited energy from the battery. In this paper, a saving energy model is proposed to guarantee minimal the energy consumption of the entire chosen path and prolong the respective node lifetime of entire path utilizing minimal power consumption of each node and extend the whole wireless network lifetime. Based on this novel saving energy model, the effect to normal channel is reduced from the energy consumption and network partition. An approximation algorithm is presented for solving this energy model considering the NPC problem about this novel energy model. Extensive simulation reveals that the proposed EA_AODV protocol is superior to the traditional AODV protocol from the perspective of saving energy and prolonging network lifework, the EA_AODV protocol validity is also verified by related simulation in this paper.
\end{abstract}

Keywords- saving energy; energy model; route; approximation algorithm;WSN network

\section{INTRODUCTION}

Wireless sensor network (WSN) is widely considered as one of the most important technologies in the twenty-first century. because sensor nodes are powered by battery and it is often very difficult or even impossible to charge or recharge their batteries, it is crucial to reduce the power consumption of sensor nodes so that the lifetime of the sensor nodes, as well as the whole network is prolonged.

Research for WSN network Saving Energy routing protocol is currently a hot topic, Several classic routing protocols are the shortest route made by IETFs MANET group, namely minimum hop routing, without considering the energy factor. However, the WSN network nodes are mostly portable devices, limited by the sized battery-powered, how to save energy nodes and prolong network lifetime as much as possible gradually become a measure of the performance of the routing protocol important indicator.

Currently, WSN energy-saving routing algorithm mainly adopts two methods. The first one has each packet sent consumed minimum energy [1-5], the second is to extend the survival time of the network as much as possible [6-10]. The first routing algorithm idea is, by finding the minimum transmission power routing so that each packet sent minimize the energy consumed, to achieve the purpose of saving energy. But it still retains the original routing algorithm problem, that is, once selected a route, it will continue using it until the data transmission is finished unless network topology changes or updates, so with minimum hop routing protocols, easy makes certain key nodes energy depletion because of the excessive use it, resulting in premature network division. The second routing algorithm idea to solve this problem protects network nodes of the less residual energy to achieve delayed network split and prolong the survival time of the network.

Since these two ideas are considered relatively one aspect, the method of this paper is the combination of these two ideas, that is combined with network lifetime and energy consumption of nodes achieving two goals condition. A new energy model is proposed is this paper to ensure that all nodes balance energy consumption and prolong the survival time of the network.

\section{A NEW ENERGY MODEL AND QUESTION FORMULATION}

\section{A. $\quad$ Service Oriented Architecture (SOA)}

According to graph theory, WSN networks can use a directed graph $G=(V, E)$ to describe, where $V$ is the set of nodes, $\mathrm{E}$ is the set of direct communication links between nodes (within communication range of each other), $|\mathrm{V}|=\mathrm{N}$, | $\mathrm{E} \mid=\mathrm{M}$. In conventional communication networks, the edge (u, $v)$ of nodes $u$ and $v$ the number of hops can be represent the hop, link capacity and the link load and so on. But here represents the energy consumption level.

In light of the signal transmission model, the received signal power can be represented $d^{-n}, \mathrm{~d}$ represents the distance between two transmission nodes within the communication range with each other. Generally, $\mathrm{n}=2$ represents the closer distance between the two nodes transmission, $\mathrm{n}=4$ is said further transmission distance. we give a formula to calculate the transmission power e as follow.

$$
P_{u, v}=\frac{\varepsilon\left\|d_{u, v}\right\|^{n}}{S_{u, v}}
$$

Where $\varepsilon$ a positive constant number, $S_{u, v}$ is the channel interference of link ( $\mathrm{u}, \mathrm{v})$ between $\mathrm{u}$ and $\mathrm{v}, d_{u, v}$ is the distance between node $\mathrm{u}$ and $\mathrm{v}, \mathrm{n}$ is 2 or 4 to take.

In this model, simplifying the relevant parameters, obtain the following formula.

This work is supported by the National Natural Science Foundation of 


$$
P_{u, v}=K d_{u, v}{ }^{\alpha}
$$

Where $\mathrm{K}$ is a positive number, $\alpha$ still takes 2 or 4 , the meaning of $d_{u, v}$ as defined above.

In fact, the network model is a directed graph $\mathrm{G}_{\mathrm{s}}=(\mathrm{V}, \mathrm{Es})$, Thai is a subset of G., $P_{t}(v)$ denotes the remaining energy of the node at the time $t$, in order to simplify the convenience, we omit the parameter $\mathrm{t}$ so that can be denoted by $P(v)$, Suppose the initial energy of the node $\mathrm{v}$ is $\mathrm{E}(\mathrm{v})$. When a node $v_{j}$ in the transmission range of node $v_{i}$ and $v_{i}$ send packets to $v_{j}$ using power $P(v)$, there is a direct link between $v_{i}$ and $v_{j}$ in Gs.. It is clear, $\left\|E_{s}\right\| \leq M, E_{s} \subseteq E$.

$P(u)$ represents the residual energy the node of $u$ at time t, $\mathrm{v}$ is directly adjacent the node $\mathrm{u}$, the ratio of the node $\mathrm{u}$ remaining energy (power consumption rate) is.

$$
\theta(u)=\frac{P(u)-K d_{u, v}^{\alpha}}{E(u)}
$$

In a directed graph $G_{s}, P$ is a directed path of directed connected link $\left(\mathrm{v}_{0}, \mathrm{v}_{1}\right),\left(\mathrm{v}_{1}, \mathrm{v}_{2}\right), \ldots,\left(\mathrm{v}_{\mathrm{l}-1}, \mathrm{v}_{1}\right), \mathrm{v}_{0}$ is the source node, $\mathrm{v}_{1}$ is the destination node. Therefore, to obtain an optimal route from $\mathrm{v}_{0}$ to $\mathrm{v}_{1}$ should meet the following two conditions.

(1) The transmission energy consumption on the path $P$ is the least, i.e. the following formula.

$$
W(P)=\operatorname{MIN}\left(\sum_{i=0}^{l-1} k d_{v_{i}, v_{i+1}}{ }^{a}\right)
$$

(2) Ensure that minimum energy consumption rate of each node in the path P maximized, that is to ensure that all nodes have the longest survival time, and then to make the longest survival time of the network.

$$
\operatorname{MAX}\left\{\operatorname{MIN}\left\{v_{i}\right\}\right\} v_{i} \in V(P)
$$

\section{THE ROUTING ALGORITHMS}

According to the energy model of the previous section, the best route required not only necessarily to meet the minimum transport energy consumption on the route path, but also to meet the longest survival time of the network. From the analysis it can be seen the problem is NP-complete problem, it is impossible to obtain the optimum results. Therefore, we construct approximate routing algorithm to ensure get a suboptimal solution..

\section{A. Approximation Algorithm Idea}

In a directed graph Gs, the weight of each edge link $\left(v_{i}\right.$, $\left.v_{i+1}\right)$ is set to double weight, repressed as the following formula.

$$
\left(K d_{v_{i}, v_{i+1}} \alpha, \theta(u)=\frac{P\left(v_{i}\right)-K d_{v_{i}, v_{i+1}}{ }^{\alpha}}{E\left(v_{i}\right)}\right)
$$

Where first item denotes the transmission energy consumption, the second term is expressed as the weight of the survival time of the corresponding node. Considering each node in the network to ensure the evenly energy consumption, the total energy consumption on route path is set to a lower bound $\beta \mathrm{L}, \beta$ is a positive number that is greater than $1, \mathrm{~L}$ is the minimum number of hops on the path, the upper bound $\mathrm{Fv}(\theta)$, denotes the maximum energy consumption from the source node to the destination node. In addition, introduce the threshold $\theta$ so that the energy consumption rate of each node is not less than $\theta, 0<\theta<1$, ensure that certain node less energy consumption.

Based on the above conditions, we can draw the algorithm model as follows.

Objective function:

$$
F_{v_{j}}(\theta)=\operatorname{MIN}\left\{F_{v_{i}}(\theta)+K d_{i, j}{ }^{\alpha}\right\}
$$

Constraints:

$$
\frac{P\left(v_{i}\right)-K d_{i, j}^{\alpha}}{E\left(v_{i}\right)} \geq \theta
$$$$
\text { (2) } F_{s}(\theta)=0
$$$$
\text { (3) } F_{D}(\theta) \leq \beta L
$$

Among them, the nodes $v_{i}$ and $v_{j}$ directly connected with each other, node $v_{i}$ send packets to its neighbors. $v_{i}$ with power $P\left(v_{i}\right)$.

\section{B. Approximation Algorithm}

given the parameter $\theta, \beta$, the function $\operatorname{FIND}\left(G_{s}, \theta\right.$, $\beta$ ) shown in Fig .1 achieves the goal that whether a certain route path from source node $S$ to destination node $D$ in Gs exists and the total transmission energy consumption of the path is less than $\beta \mathrm{L}$, while the energy consumption rate for each node is not less than $\theta$.

$$
\begin{aligned}
& \operatorname{FIND}(G s, \theta, \beta) \\
& F_{S}(\theta) \leftarrow 0 ; \\
& \text { Length_Path_Nodes }[S] \leftarrow S ; \\
& \text { For all } v \in \mathrm{V}-\{S\} \text { do } \\
& \quad F_{v}(\theta) \leftarrow \infty ;
\end{aligned}
$$

Endfor

For $\mathrm{i} \leftarrow 1$ to $N-1$ do

$$
\text { For each }(\mathrm{u}, \mathrm{v}) \in E s \text { do }
$$

$$
\begin{aligned}
& \text { If } \frac{P(u)-K d_{u, v}^{\alpha}}{E(u)} \geq \theta \text { and } \\
& F_{v}(\theta) \geq F_{u}(\theta)+K d_{u, v}{ }^{\alpha} \\
& \text { Then } F_{v}(\theta)=F_{u}(\theta)+K d_{u, v}{ }^{\alpha}
\end{aligned}
$$




\begin{tabular}{|l} 
Length_Path_Nodes $[u] \leftarrow v ;$ \\
Endfor \\
Endfor \\
If $F_{D}(\theta) \leq \beta L$ \\
then return true; \\
Else return false; \\
Endif $\quad$
\end{tabular}

Figure 1. function $\operatorname{FIND}\left(G_{s}, \theta, \beta\right)$

Based on the functions $\operatorname{FIND}\left(G_{s}, \theta, \beta\right)$, the function Find_Approximation_Path shown in Fig .2 determine a less energy consumption path, achieves the near optimal purposes.

Find_Approximation_Path $(G s, \varepsilon)$

Min_level $\leftarrow \varepsilon ; \quad / /$ the minimum bound of $\theta$

Max_level $\leftarrow 1 ; \quad / /$ the maximum bound of $\theta$

While $\left(M a x \_l e v e l-M i n \_l e v e l>\varepsilon\right)$ do

$$
\theta \leftarrow \frac{\text { Max_level }+ \text { Min_level }}{2} ;
$$

If $\operatorname{FIND}(G s, \theta, \beta)$

Then Min_level $\leftarrow \theta$;

Else Max_level $\leftarrow \theta$;

Endif

Endwhile

Figure2. function Find_Approximation_Path (Gs)

\section{SIMULATION}

NS2 is an experimental simulation platform in this paper, based on a modified AODV protocol draw EA_AODV, compare the two aspects relative performance of the energy consumption and the network lifetime. The required network topology of experiments was generated by the NS2 setdest tools. The running rate and the initial positions of the nodes are randomly set during the entire simulation time to simulate the random running scene. Regional scene for the whole experiment is $500 \mathrm{~m} \times 500 \mathrm{~m}$, the simulation time is $900 \mathrm{~s}$, the maximum running rate of nodes is $15 \mathrm{~m} / \mathrm{s}$. The number of nodes in the simulation scenarios is 25 , the initial energy is 10 , 20,30 and 40 energy units respectively, residence time is $0,20,120,600,900$ respectively. 15 kinds of random experimental topology are generated, each case correspondes five kinds. The final data is of the average value of the five kind topology data. Random data flow between nodes is set by cbrgen tool, The udp stream is generated by randomly generated 20 sender to 20 recipient, Each CBR packet is 512 bytes, 4 packets are transmitted per second, bandwidth is of $2 \mathrm{M}$, Transmitting radius of nodes is of $250 \mathrm{~m}$. Other parameters used in the experiment is as follows: channel and wireless models is two-ray ground reflection model, MAC layer uses the IEEE 802.11 DCF (Distributed Coordination Function).

Fig .4 to Fig .6 show, at different initial energy and residence time situations, the total energy consumption and the network survival time of the two protocols. Performance parameters of the two comparison are under the following description.

(1) Energy consumption: The total of the energy consumption of all nodes from 0 s and 900 s simulation time.

(2) The network lifetime: The death time of the first node in the network.

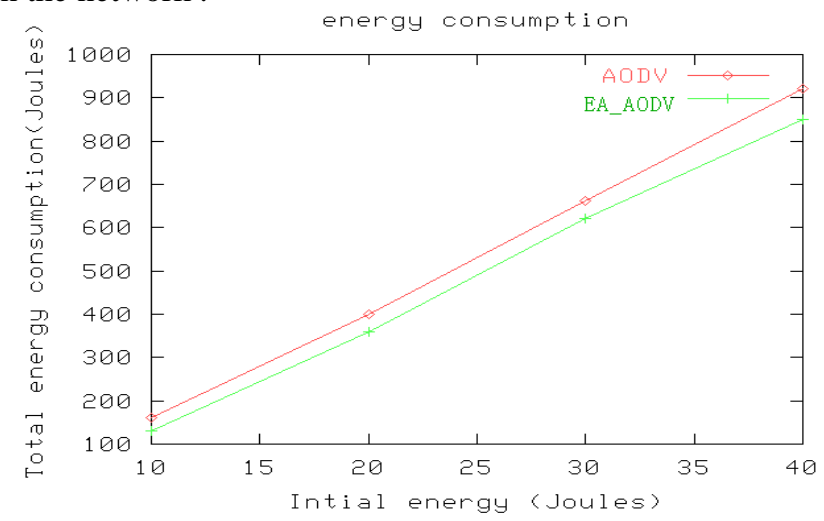

Figure 4. Initial energy and total energy consumption

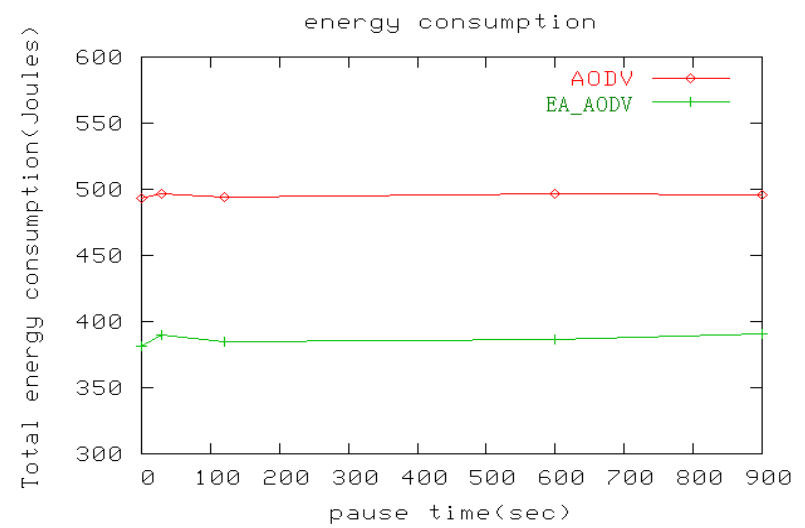

Figure 5. Residence time and the total energy consumption

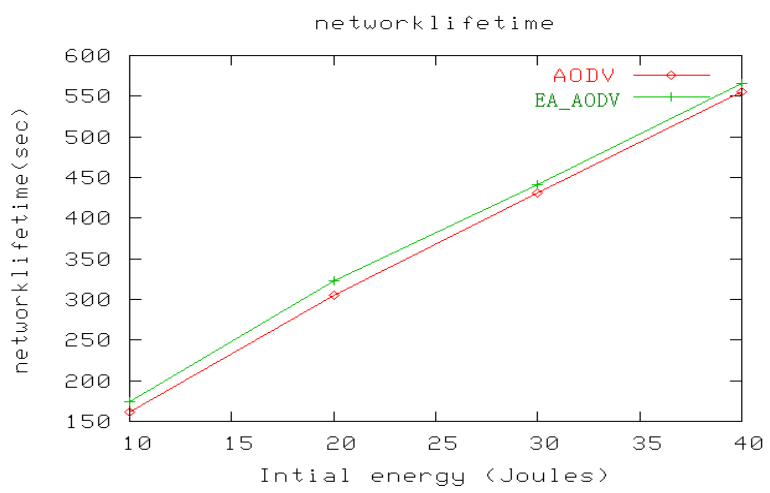

Figure 6. Initial energy and network lifetime

As can be seen from Fig .4, the energy consumption of the protocol EA_AODV slightly smaller. Because the protocol when looking for a suitable route, each node takes into account the energy consumption, but also consider the rate of energy consumption, so that in the case of satisfying the above two factors, to reduce the total energy consumption. Fig .5 shows the node under different residence time, energy consumption and less taught EA_AODV protocol, which is mainly due to the increased energy efficiency of the policy and adding the residence time, thereby reducing the consumption 
of excessive energy nodes. Fig .6 reflects the agreement in EA_AODV different initial energy, the lifetime of the network longer, due to our algorithms and models, to ensure minimum energy consumption rate of each node of the premise, to avoid excessive energy consumption node, thereby increasing the survival time of the network. This effect is also obtained from Fig .7, the performance. Therefore, from different initial energy and residence time of two terms, EA_AODV agreement than has been significantly improved performance on AODV. However, the improvement of this paper is built on four possible routes break situation, so find other situations that may arise, quickly establish alternative routes and carry out other aspects of our performance comparison in another direction for future research.

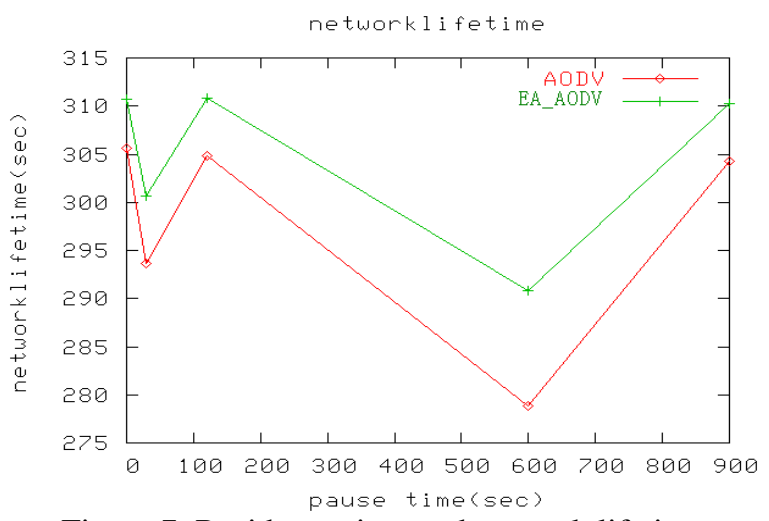

Figure 7. Residence time and network lifetime

\section{Simulation}

WSN network routing algorithm based on limited energy is a hot topic. In the current research background, consider the node energy consumption of two aspects, to ensure the minimum total energy consumption on the path, while to prevent excessive energy consumption of each node to satisfy a long lifetime of each node, so that the survival time of the network can be extended. The issue meeting these two objectives is a NP problem, therefore propose an approximation algorithm to find an approximate optimal path. The protocol is simple, relatively easy to achieve. Future studies may add some related QoS metrics to expand the protocol, so that there are good theoretical guidance in realtime multimedia applications in WSN network.

\section{ACKNOWLEDGMENT}

This work is supported by the National Natural Science Foundation of China under grant number 61262087 and 61262089, Key Project of Xinjiang College Teachers Research(Project No. XJEDU2012I09) and Xinjiang University PhD Scientific Research Fund(Project No. BS110127). The authors wish to thank many referees for their suggestions on improving this paper.

\section{REFERENCES}

[1] K. Akkaya and M. Younis, "An Energy-Aware QoS Routing Protocol for Wireless Sensor Networks," in the Proceedings of the IEEE Workshop on Mobile and Wireless Networks (MWN 2003), Providence, Rhode Island, May 2003.

[2] S. Lindsey, C. S. Raghavendra, and K. M. Sivalingam, "Data gathering algorithms in sensor networks using energy metrics", IEEE Transactions on Parallel and Distributed Systems, vol. 13, no. 9, Sept. 2002, pp. 924935.

[3] W.R. Heinzelman, A. Chandrakasan, and H. Balakrishnan, "Energyefficient Communication Protocol for Wireless Microsensor Networks", in IEEE Computer Society Proceedings of the Thirty Third Hawaii International Conference on System Sciences HICSS '00), Washington, DC, USA, Jan. 2000, vol. 8, pp. 8020.

[4] Ossama Younis and Sonia Fahmy" Heed: A hybrid, Energy-efficient, Distributed Clustering Approach for Ad-hoc Networks", IEEE Transactions on Mobile Computing, vol. 3, no. 4, Oct.-Dec. 2004, pp. 366-369.

[5] DeyingLi, ZewenLiu, YiHong " Minimum energy multicast/broadcast routing with reception cost in wireless sensor networks", Theoretical Computer Science, vol. 497, no. 4, 2013, pp. 173-180.

[6] GUPTA N, DAS S R.Energy-Aware On-Demand Routing for Mobile Ad hoc Networks[EB/OL].http //crewman.uta.edu/ choi/energy.pdf,2005.

[7] Yin, SY, Lin, XK.Multipath minimum energy routing in Ad hoc network.ICC 2005 : IEEE INTERNATIONAL CONFERENCE ON COMMUNICATIONS, VOLS 1-5 : 3182-3186 2005.

[8] Liang, WF. Minimizing energy and maximizing network lifetime multicasting in wireless Ad hoc networks. ICC 2005 : IEEE INTERNATIONAL CONFERENCE ON COMMUNICATIONS, VOLS $1-5: 3375-3379,2005$.

[9] Deying Li,Qin Liu,Xiaodong Hu,Xiaohua Jia. Energy efficient multicast routing in ad hoc wireless networks. Computer communications, 2007,30(6):3746-3756.

[10] RODOPLU Volkan, MENG Teresa H Y. Minimum energy mobile wireless networks. IEEE Journal on Selected Areas in Communications, 1999, $17(8) \quad: \quad 1-17$. 